\title{
A simulation game for nonlinear control theory education*
}

\author{
Rune Haugom $^{2}$ Ole K. Solbjørg ${ }^{1}$ Kristin Y. Pettersen ${ }^{2}$ Tor I. Eikaas ${ }^{3}$ \\ ${ }^{1}$ Social Research Ltd, Program for learning with ICT, Norwegian University of Science and Technology, N-7491 \\ Trondheim, Norway. \\ ${ }^{2}$ Department of Engineering Cybernetics, Norwegian University of Science and Technology, N-7491 Trondheim, \\ Norway. \\ ${ }^{3}$ Cyberlab Org. AS, Trondheim, Norway.
}

\begin{abstract}
In this paper a computer game implemented as an applet for use in a graduate course in nonlinear control theory is described, and the learning value of the game is substantiated using different learning theories and theories related to ICT. The case study of the applet is a mass-damper-spring system with a nonlinear spring characteristic, and a game with a highscore list has been made out of the system to stimulate student competition and motivation.
\end{abstract}

Keywords: Learning theories, simulation, linear/nonlinear control, computer game, graduate course

\section{Introduction}

The use of simulations of physical systems in higher education is an exciting field in rapid development. New technologies are developed and implemented, and the pedagogical use of these is piloted. Nowadays, computers and the Internet are used more and more in the everyday life of the students, and they expect that multimedia is also used for educational purposes. Computer games have also been a part of many students' childhood and still many think that computer games are quite fun. For instance popular Java games that can be found everywhere on the Internet is a proof of that. Therefore, including computer games for higher educational purposes might be quite fortunate. The development of the simulations should be done in combination with relevant pedagogical theories to get the best possible motivation and learning effect. In this paper a simulation game implemented as a Java applet

\footnotetext{
*Published at 7th IFAC Symposium on Advances in Control Education, Madrid, Spain, June 21-23, 2006.
}

for use in a graduate course in nonlinear control theory is described, and the learning value of this applet is substantiated using different learning theories and theories related to Information- and Communication Technology (ICT). The main purpose of this article is not to describe different control strategies for the system, but to describe the means supported by learning theories that have been used to increase the learning value of the interactive simulation. People who do not have an advanced background in control theory should therefore be able to understand the most of this paper.

A mass-damper-spring system is the case study of the applet, but as a contrast to the traditional spring following Hooke's law, the spring characteristic is nonlinear, which makes this a relevant case for nonlinear control theory. The applet has been integrated in the PIDstop simulation platform, which is a web-based educational system for technology and science developed for the business of automation (Foss and Eikaas (2006) and Eikaas et al. (2005)). By using a Java applet it 
is straightforward to visualize the behaviour of physical systems like a mass-damper-spring system. What is needed for this is the instant position of the system and that is easily available in PIDstop since it has a built-in numerical solver for solving the differential equations describing the system dynamics.

For the past few years several applets for both graduate and undergraduate studies at the Norwegian University of Science and Technology have been developed, and more and more teachers have brought these applets into their courses for attractive demonstrations of important concepts. The interdisciplinary collaboration this represents benefits both the pedagogical research field and targeted development of technologies. The developments in ICT have afforded increasingly advanced possibilities for utilization in education, and it seems to some degree to have followed a trajectory in pedagogical theory from the behaviourist approach, through cognitivism and constructivism, to socio-cultural learning theories where ongoing research in the field focuses on learning communities. To place the use of simulations in a pedagogical context, a brief account of relevant learning theories are given. As the simulators in this case are not developed for collaboration through the Internet, socio-cultural learning theories are not elaborated. After that it is shown how the applet has been developed to offer a rich learning experience as required according to constructivist learning theories. This paper is organized as follows: In Section 2 the learning theories are presented and in Section 3 simulations and games for learning purposes are described. Then a description of the applet is given and finally some conclusions are drawn.

\section{Learning theories}

In behaviourist learning theory (central theorists are Edward Lee Thorndike (1874-1849), John B. Watson (1878-1958) and Burrhus Fredric Skinner (1904-1990), see for instance Hergenhahn and Olson (2004)), learning is seen as the process of reinforcing desired responses through repeated stimulus-response cycles. In this process correct responses are rewarded, and there may also be a punishment for incorrect responses. For certain learning material, this approach may be very effective. Skill and drill programs (more popularly referred to as "drill and kill") are successfully used in learning factual knowledge such as vocabulary training, memorising multiplication tables and learning geography. It is widely used in children's games for learning shapes, colours, letters and so forth. The "reward" given for correct responses may range from a numeric score to smiley faces or fanfares; what is most important is that the feedback is perceived as a reward. When computers were first introduced in education, the processing power did not allow for sophisticated graphics or demanding simulation algorithms. Programs for drilling specific knowledge in the form of eliciting correct responses are fairly simple to both program, administer and use, yet they give a good learning effect when used correctly.

In cognitivistic learning theory (central theorists are John Dewey (1859-1952), Jean Piaget (1896-1980) and Lev Vygotsky (1896-1934), see for instance Hergenhahn and Olson (2004)) the learner is seen as more active in processing input than in behaviourist learning theory. The learner builds on current knowledge to incorporate new knowledge as she/he develops new cognitive structures. The learning material needs to be on a level that allows the learner to connect this to existing knowledge structures, and new material must be structured in a manner that facilitates the learner's acquisition. Self-regulated learning is a model often used when developing ICT-based learning programs. (See for instance Zimmerman (1998)) Here, the learning material is presented in a clear structure where the learner is allowed to move on to the next level after mastering a learning task.

Based on the work of cognitivist learning theory, constructivist learning theory Hergenhahn and Olson (2004) has evolved. Constructivist learning theories see the learner as active in seeking knowledge and building her or his understanding of objects and processes. Facilitating ICT-based learning following this approach means providing the learner with rich learning experiences. This will allow for the learner to actively experiment through building on existing knowledge, reflecting on this, and if necessary seek more information to reach their learning goals and build further knowledge.

\section{Simulations and games for learning purposes}

As computer technology has developed, it has allowed for more complex and advanced representations of learning material. Thus, ICT has been increasingly more suited for applying constructivist approaches to learning. (For literature reviews in the area of ICT and learning, see for instance http://www.nestafuturelab. org/research/lit_reviews.h tm.) Multimedia presentations are vastly more powerful in providing learners with the rich learning environment on his or her quest to construct the desired understanding of complex objects and processes. In addition, Internet technology 
facilitates the search for further information needed; either within a site provided by the teacher, containing the necessary material in a more or less defined structure, or on the Internet.

Visualisations allow for the representation of processes that are not readily available to the learners. These processes may be so expensive, large, fast or even dangerous, that it is not possible to provide the learners with first hand experience. Powerful learning materials as they may be, mere visualisations do not provide the ultimate learning experience where one is given the possibility to actively experiment with the process at hand. Simulations are a further development providing these possibilities. Simulations may be defined as visualisations of processes, with the added possibility for changing parameters and observing the result. Also, the underlying parameters, forces and constants may be displayed on the screen and updated in real-time together with the visualisation, thus establishing a connection between the theoretical concepts and the process.

When developing simulations there are several aspects to take into consideration. One may start with defining what the learning outcome should be for students using the simulation. On the other side, it is in fact possible for one simulation to be used in learning on different levels. By removing parameters, or automating some settings, a highly advanced simulation may be used for learning more fundamental aspects of a process, even if the simulation offers the possibility to modify every single parameter inflicting the process. Also, the simulation may be used only to present a concept without emphasis on the mathematical or even some of the underlying theoretical aspects. In addition it may be possible to incorporate certain simulations in other subject matters than those they are designed for, as certain core processes are highly relevant to several adjacent subjects.

Another starting point when developing simulations may be the form of representation. One may decide to present for instance a physical mechanism, like in this case a mass-damper-spring system, in its most simple form. However, one may improve the students' possibility for grasping the concept presented by relating the simulation to real life settings - theoretically speaking, by relating it to the students' existing knowledge, allowing them to reflect and build on this.

To increase motivation, it is also possible to find a metaphor that is suited for developing the simulation into a game. Little research is conducted on the use of computer games in higher education teaching. Yet, earlier experiences with using the PIDstop simulation platform at the Norwegian University of Science and Technology indicate that the students appreciate the novelty and variation offered by this. The result is that they dedicate more time to exercises based on these simulations, presumably resulting in better learning.

There are a number of factors to consider to turn a simulation into a game that is motivating, and at the same time maintains academical credibility and offers a realistic setting that supports the students' process of reflection and knowledge development. Also, there is a limitation on resources. Computer games are increasingly realistic, bordering on cinematic quality. Higher education institutions will never be able to compete with these games in playability, or finesse in graphics and audio. Still, it is possible to make stimulating and interesting games simulations in $2 \mathrm{D}$ with simple audio effects. All the youngsters who spent countless hours playing games on their ZX Spectrum and Commodore 64 machines during the 80's are proof of that, and still children are delighted by the many simple Java and Flash games available for free on the Internet. Important factors to develop successful simulation games are:

- The metaphor or context chosen for the game should be easily recognizable.

- The role of the process being simulated must be obvious in the game.

- The game should comply to physical laws (which is not necessary in traditional computer games).

- The goal of the game should not be achievable through trial and error.

- Sound effects are not vital, but should be incorporated to prevent the game from appearing too simplistic.

- The use of humour is expected to increase motivation in students in higher education.

- There should be several levels. This will in itself give the student valuable feedback on her or his progress and increase motivation.

- Rankings, using time or points as scores, gives a competitive edge that will increase the probability that students try several times.

\section{DuckMaze}

Duckmaze is a simulation game developed for a graduate course in nonlinear control theory. An important part of this course is analysis of stability and design of 
controllers for nonlinear systems using Lyapunov techniques. Other important techniques are input-output linearization, backstepping and passivity theory. The intention of the applet is to give the students some tuning experiences for nonlinear controllers. A massdamper-spring system with nonlinear spring force is the case study, and this system has been chosen because it is a system where nonlinear control theory enables better control than the linear control theories that the students have learned in their undergraduate courses in linear control theory, thus creating motivation for the course in nonlinear control theory. It is also quite simple both to analyse and to visualize, and it is possible to make an attractive game out of it that looks like other popular 2D games, which can be found everywhere on the Internet. The simulation game is presented to the students in the context of a mandatory exercise, which in addition to the game contains problems in nonlinear analysis and control design related to the game. This prevents students from only playing with the game without learning anything. The problems require the students to analyse mathematical expressions for the system and to develop nonlinear controllers using design methods from the course syllabus. This kind of problems has been given to the students for several years. The new aspect is that now they also have the ability to test the controllers on a simulator of the mass-damper-spring system. This also gives them some tuning experiences of the nonlinear controllers. Simulations from earlier courses have usually involved using simulation programs like Matlab/Simulink, which produce state plots as a function of time. In this applet an actual graphical representation of the system is given, and this will hopefully make the simulation more attractive. The applet has two modes, a practice mode and a game mode where the game mode has been included to catch the students' interest. Hopefully, this will make them spend some time with it. DuckMaze is presented at the web page http://www.pidstop.com/index.php? $r_{-} i d=214$.

\subsection{Practice mode}

Figure 1 shows a picture of the practice mode of the simulator. In this mode the user can test the massdamper-spring system by using one of the built-in controllers described below, by controlling the system manually, by using a user-defined controller or by using a demo controller. The user can also change the system parameters and disturb the system by an external force and observe how the system behaves. Parameters that can be changed are the mass of the system, the damping constant, the spring constants and the initial values of the position and the velocity of the system. The intention of this mode is to give the user an opportunity to test the system and the controllers without having all the additional graphics that is included in the game mode.

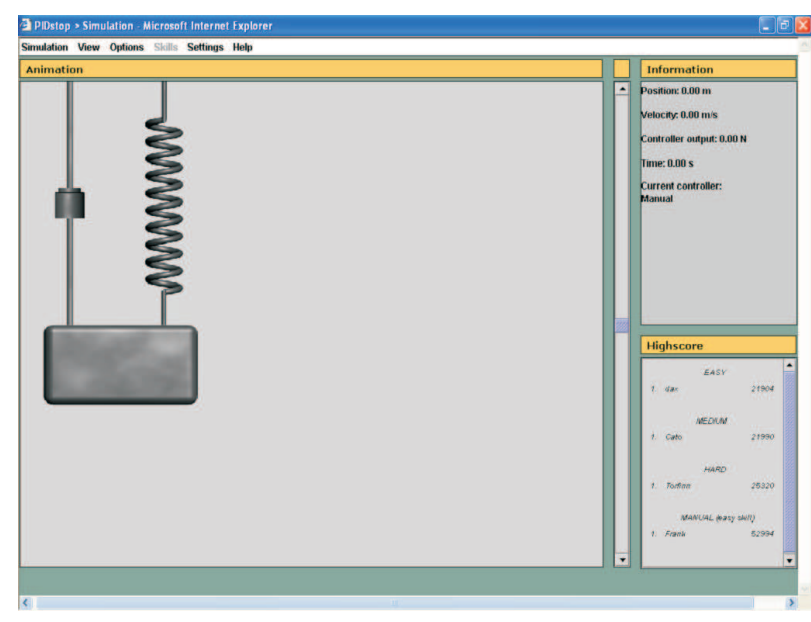

Figure 1: The practice mode.

\subsection{Game mode}

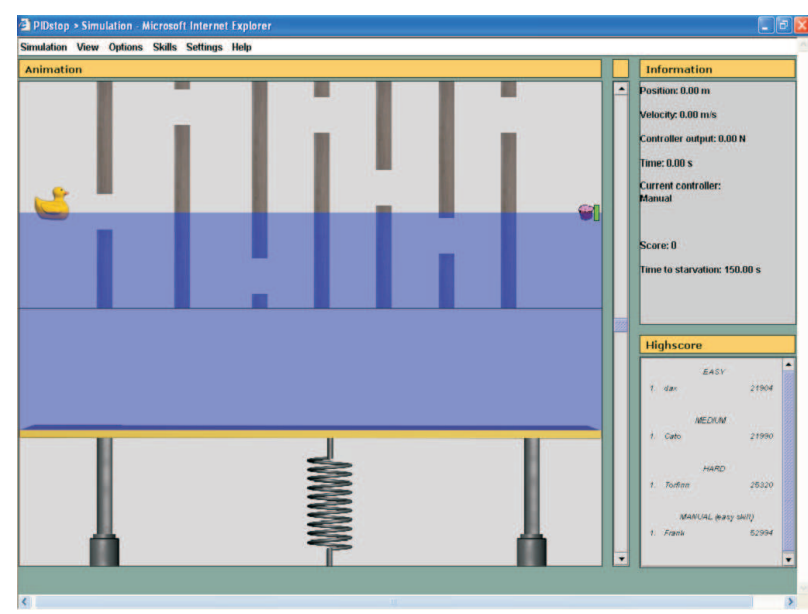

Figure 2: The game mode.

A screenshot of the game mode is shown in Figure 2 and the mass-damper-spring system can be easily recognized at the bottom of the figure. This is important as explained in Section 3. Water has been placed on the top of it and the idea is to control the position of the duck on the water surface by controlling the position of the mass-damper-spring system. The position can either be controlled manually by using the scrollbar to the right of the animation panel in Figure 2 or by using a controller. The game scenario is as follows: 
A duck is very hungry and needs food really fast. It is very lucky as there is a blueberry muffin to the right of the screen. The problem is that between the duck and the muffin there are a lot of obstacles that the duck must get through. Your job is to control the position of the water surface so that the duck can swim through the small openings in the obstacles. As explained at the end of Section 3, the game should have several skill levels; DuckMaze has three:

Easy. At this skill level the user knows the exact values of the system parameters and no disturbances are present. To prevent the user from making an indefinitely fast controller, the controller output has been limited to $\pm 40 N$. By using for instance a properly tuned nonlinear input-output linearization controller, the system performance will be better than what is possible to achieve with a linear controller. This is important to increase the students' motivation.

Medium. At this level the exact values of the system parameters are also given to the user. However, this time dynamite cubes keep falling down from the sky and the explosion results in an impulse force disturbing the system. This requires a faster controller than at the easy skill level since the duck needs to get into position to swim through the obstacle before the next dynamite cube explodes. To prevent the user from making an indefinitely fast controller, the controller output is as before limited to $\pm 40 N$. Also, at this level it is possible to achieve a better result by using a nonlinear controller instead of a linear one.

Hard. This level does, as skill level medium, contain exploding dynamite cubes. The interval between the explosions are, however, smaller and an even faster controller is required. On skill level easy and medium the exact values of the system parameters were given to the user but at this level this is not the case. In addition, the water that lies at the top of the mass-damperspring system is given a mass that is unknown to the user. Therefore, both linear and nonlinear controllers are dependent on integral action to achieve a perfect static performance. At skill level easy and medium it was possible to improve the system performance by using a nonlinear controller instead of a linear one. However, simulations show that this is not the case on this level. To prevent the user from making an indefinitely fast controller, the controller output has been limited. Since the water is given a mass on this level, more power is needed to lift the mass-damper-spring system. Therefore, the saturation limit is increased to $\pm 100 N$.

\subsection{Simulator features}

Easy-to-use user-interface. It is important that the user does not spend to much time figuring out how the applet works. Therefore help pages on how to use the simulator are available as well as a description of the system model and the symbols used.

Change system parameters. In the practice part of the simulator it is possible to change the system parameters like the mass of the system, the damping constant, the spring constants and the initial conditions for position and velocity. Process noise in the shape of a constant disturbing force can also be added. In addition, it is possible to set a reference value and limit the controller output by using a saturation element.

Competition/Highscore list. As explained in Section 3, the game should include some sort of ranking mechanism as this will stimulate for competition of making the best controller among students. In DuckMaze there is a highscore list for each skill level and one for the manual mode. The score is based only on the time it takes to guide the duck through the maze. If you are familiar with nonlinear controllers and manage to outline the correct control laws and in addition is good at tuning the controller, the duck will faster get through the maze and you are credited by a higher score.

Interaction. In addition to developing and tuning the controllers, the user has to choose the reference values that makes it possible for the duck to swim through the next opening. This choice could have been made automatically, but is left to the user in order to increase the interaction features of the simulation game.

Help placing the correct reference value. Since the score in the game mode should be based on knowledge in control theory (not on finger skills), it should not be difficult to choose the reference described above. A socalled snap-function has been included in the program for this purpose. This involves a blue cross that tells the user where to put the reference signal and it gets visible when the mouse cursor is in the vicinity of the middle of the openings. Pressing the mouse button at this time will automatically set the position of the mass-damper-spring system to the position of the blue cross.

Graphical representation and real time plots. The simulator provides both a real-time visualization of the mass-damper-spring system as well as real-time plots of the system states and the controller output. This gives a better understanding of the traditional time plots since it is possible to compare these with the visual drawing of the system. Traditional plots in this context means that the plots are similar to the time plots that the students meet in the courses and the textbooks. It is also possible to save the plot data to a file that can easily be imported into Matlab.

Five built-in controllers. Five controllers have been implemented in the program. These are:

- Linear state feedback controller. This is a standard linear state feedback controller given by $u=$ 
$-k_{1} x_{1}-k_{2} x_{2}-k_{3} x_{3}$ where $x_{1}$ is the position of the system, $x_{2}$ is the velocity of the system and $x_{3}=\int\left(x_{1}-x_{1 d}\right) d t$ where $x_{1 d}$ is the desired position. $k_{1}, k_{2}$ and $k_{3}$ are the controller gains and can be set by the user.

- PID controller. This is a standard PID controller given by $u=k_{p} e+k_{i} \int e d t+k_{d} \dot{e}$ where $e=x_{1}-$ $x_{1 d}$ and $x_{1 d}$ is the desired position. $k_{p}, k_{i}$ and $k_{d}$ are the controller gains and can be set by the user.

- User-defined controller. Controllers other than the ones given in this section can be implemented by downloading a template (Java file) and specify the control law. How to do this is described in a tutorial given at the web site of the applet. It is really easy, and the user does not need to know Java at all. From a pedagogical point of view, it is more stimulating to have the ability to define a user-defined controller rather than being forced to choose a predefined one.

- Manual controller. This controller makes it possible for the user to use the scrollbar to the right of the animation panel in Figure 2 to manually control the system. The force acting on the system is determined by the position of the scrollbar. This controller has mainly been included to give the users an idea of how difficult it is to control the system manually.

- Demo controller. A combination of an inputoutput linearization controller and a backstepping controller has been implemented as a demo controller. At skill level easy and medium the input-output linearization controller is used and at skill level hard, the backstepping controller is used. The demo controller provides the user with a functional controller so that people who do not have a background in control theory can see how it works.

\subsection{Gaming effects}

One of the main points of the applet is to let students get some experiences tuning a nonlinear controller that has been developed in the exercises. To make them spend quite some time on this, it is very important that the game is attractive and several effects have been included in the game to achieve this goal.

Sounds. As explained at the end of Section 3, sound effects should be included in a game, and in DuckMaze three different sounds are being used. Firstly, as the duck is blocked by an obstacle it starts quacking and this goes on until the duck is in position to move on to the next chamber in the maze. Secondly, on skill level medium and hard, there is an explosion sound as the dynamite goes off and thirdly, as the game finishes, a fanfare is played.

Highscore list. This stimulates for student competition of making the best controller and increases the probability that students try several times as explained in Section 3.

Limited time. The time available to guide the duck through the maze is limited. This has been done by purpose to introduce the I-need-to-hurry-or-else-theduck-will-die-feeling many people get as the time starts to run out.

The use of humour. The hero of the game is a yellow bathing duck. Any dead object could have been used instead of the duck, but the duck makes the game a bit cartoon-like. As explained in Section 3 this is expected to increase motivation.

Interaction with the user. A 'game' where the user just presses a play button and watches the output is not a game; there has to be some sort of interaction between the user and the game to make it interesting. In educational purposes where better knowledge in control theory should give a better score, this is quite unfortunate as finger skills come into question. In this applet however, the issue about finger skills should not be a big problem. It takes several seconds for the duck to leave one room in the maze and enter the next one, and the user has all this time available to click the mouse button to set the reference point for the next position of the duck.

\section{Conclusions}

In this paper a simulation game for use in a course in nonlinear control theory has been described and the learning value of the game has been substantiated by different learning theories and theories related to ICT. The game is presented at http://www.pidstop.com/ index.php? $r_{-} i d=214$. An interesting approach to further development in the area would be to increase focus on collaborative learning. Both existing technology and pedagogical theory offers possibilities for this, and technologists and educationalist should elaborate on this in close cooperation.

\section{References}

Eikaas, T. I., Foss, B. A., Solbjørg, O. K., and Bjølseth., T. Game-based dynamic simulations supporting technical education and training. 2005. ICL 2005, September 28-30, 2005, Villach, Austria. 
Foss, B. A. and Eikaas, T. I. Game play in engineering education - concept and experimental results. 2006. The International Journal of Engineering Education, Volume 22, no. 5, 2006.

Hergenhahn, B. and Olson, M. H. An Introduction to Theories of Learning. Prentice Hall, 7th edition, 2004.

Zimmerman, B. J. Developing self-fulfilling cycles of academic regulation: An analysis of exemplary instructional models. In D. H. Schunk, B. J. Zimmerman (Eds.), Self- Regulated Learning: From Teaching to Self-Reflective Practice. New York: Guilford Press, 1998 pages 1-19. 